COMMENT. Under different terminologies, this syndrome has been described in various case series, and was first reported in Brain (1961;84:680-708) as "acute encephalopathies of obscure origin" by Lyons, Dodge, and Adams. "Lyons-DodgeAdams syndrome" would be a suitable eponym. Further research concerning the etiology suggested by the current authors includes immune and nonimmune mechanisms (eg.channelopathies, antibodies against ion channels and receptors, and infectiontriggered alterations of receptor expression). A special issue on acute encephalopathy/encephalitis in childhood appears in Brain Dev June 2010.

\title{
ACUTE LIMBIC ENCEPHALITIS IN JAPANESE CHILDREN
}

Clinical, laboratory, and radiographic findings in 14 cases (median age 10 years; range 4-14 yrs) of nonparaneoplastic limbic encephalitis were analyzed by researchers at the National Center of Neurology and Psychiatry, Kodaira, Japan. Infectious febrile illness preceded onset by $0-9$ days in 12 patients. Seizure $(n=10)$ was the most common initial symptom, consciousness was impaired in 5 , and 3 presented with psychiatric symptoms. All patients developed impaired consciousness, short-term memory loss occurred in 12, and psychiatric symptoms were common (emotional lability $(\mathrm{n}=7)$, irritability (7), hallucinations (4), aggression (4)). Ten patients had all 3 signs of limbic encephalitis (short-term memory deficit, limbic seizures, and psychiatric symptoms). Other symptoms included movement disorders (4), and dysarthria (2). CSF showed mild to moderate pleocytosis in 8 patients, and elevated protein in 4. EEG showed slowing. MRI revealed signal abnormalities in hippocampal or amygdaloid formations in 9 . Herpes simplex virus antibody or PCR was negative in all. Anti-voltage-gated potassium channel antibody was negative in one patient. Other autoantibodies associated with limbic encephalitis were not examined. Ten patients received immunomodulatory treatment (corticosteroids, iv immunoglobulin, and plasmapharesis), and 12 were given prophylactic acyclovir. Overall prognosis was favorable: 10 recovered with normal IQ; only one had severe cognitive impairment. Neurological sequelae included epilepsy in 5, psychiatric disorder (3), and memory impairment (3). (Sakuma H, Sugai K, Sasaki M. Acute nonparaneoplastic limbic encephalitis in childhood: a case series in Japan. Pediatr Neurol Sept 2010;43:167-172). (Respoñd: Dri Sakuma. E-riail: sakumah@ncnip.go.jp).

COMMENT. The authors conclude that childhood limbic encephalitis differs from that in adults. Whereas initial symptoms in children are principally acute seizures and impaired consciousness, adults present with subacute memory impairment or psychiatric symptoms, and most cases of limbic encephalitis in adults are associated with neoplasm. Antecedent infection may cause limbic encephalitis in children through a secondary autoimmune response. This study was limited by the lack of autoantibody tests. Age related differences in symptoms of limbic encephalitis in children and adults will require investigation through immunological, including humeral and cellular immunity.

Temporal progression of non-paraneoplastic NMDA antibody encephalitis in adults, studied in 35 European patients, found 10 ( 8 female) aged 17-44 years (median $25.5 \mathrm{yrs}$ ) 
had two to four relapses within 3 months and 6 years following improvement after the initial episode. These patients had received minimal or no immunotherapy for the initial illness. Good clinical outcome correlated with decreased NMDA receptor antibody levels and were associated with early immunotherapies. Progression in relapse cases was in two stages, initial neuropsychiatric symptoms and seizures, followed later by onset of movement disorders, impaired consciousness and dysautonomia. Early features are associated with CSF lymphocytosis, and later features with appearance of oligoclonal bands. (Irani SR et al. Brain 2010;133:1655-1667).

\section{MMR-VARICELLA VACCINE AND RISK OF FEBRILE SEIZURES}

Researchers from the Kaiser Permanente Vaccine Study Center, Oakland, CA, other centers in the US, and Centers for Disease Control and Prevention, Atlanta. GA, using 2000-2008 Vaccine Safety Datalink data, compared the risk of febrile seizures among children aged 12 to 23 months after MMRV and separate MMR + varicella vaccines. Analyses included Poisson regression and supplementary regressions incorporating chart-review results. Seizure and fever incidence significantly peaked 7 to 10 days after vaccination with all measles-containing vaccines but not after varicella vaccination alone. The relative risk of seizures was $7.6(\mathrm{P}<0.0001)$ after $\mathrm{MMRV}$ combined vaccination, $4.0(\mathrm{P}<0.0001)$ after $\mathrm{MMR}+$ varicella separate vaccinations, and $3.7(\mathrm{P}<0.0001)$ after MMR vaccination alone. No seizure peak occurred after varicella vaccination alone. Rates for seizures during days 7 to 10 were $84.6 / 1000$ person-years after MMRV, 42.2 seizures per 1000 person-years after MMR + varicella, and 26.4/1000 after MMR alone. Unadjusted rates were 8 times higher for MMRV and 4 and 3.5 times higher for MMR + varicella and MMR vaccination alone, respectively. Outpatient fever visits sharply increased during days 7 to 10 , most actively after MMRV. The proportion of children with post-vaccination febrile seizures with a history of seizures was similar for MMRV $(16 \%)$ and MMR + varicella vaccines $(22 \%)[\mathrm{P}=0.21]$. Of those with febrile seizures, family history was positive for seizures among $30 \%$ of MMRV recipients vs $29 \%$ of MMR + varicella vaccines $(\mathrm{P}=0.90)$. The use of MMRV vaccine instead of separate MMR + varicella vaccines approximately doubles the risk for fever and febrile seizures. Providers who use the combination vaccine should notify parents of this increased risk of febrile seizures. (Klein NP, Fireman B, Yih WK, et al. Measles-mumpsrubella-varicella combination vaccine and the risk of febrile seizures. Pediatrics July 2010;126:e1-e8). (Respond: Nicola P Klein MD PhD, Kaiser Permanente Vaccine Study Center, 1 Kaiser Plaza, Oakland, CA 94612. E-mail: nicola.klein@kp.org).

COMMENT. The risk of fever and febrile seizures is increased 7 to 10 days following all measles-containing vaccines, and the risk after the combination MMVR vaccine is double that associated with separate MMR + varicella vaccines. Varicella vaccine alone does not increase febrile seizure risk.

Diphtheria-tetanus acellular pertussis vaccine (DTaP) in children aged 6 weeks to 23 months showed no increased risk of seizures within 0 to 3 days after vaccination, in a retrospective study from 1997 through 2006 conducted by 7 managed care organizations and the Vaccine Safety Datalink Team using risk-interval cohort and self-controlled case 